\title{
Genetic characterization of Toxoplasma gondii from pigs from different localities in China by PCR-RFLP
}

\author{
Hai-Hai Jiang ${ }^{1}$, Si-Yang Huang ${ }^{1}$, Dong-Hui Zhou' ${ }^{1}$ Xiao-Xuan Zhang ${ }^{1,4}$, Chunlei Su ${ }^{1,3}$, Shun-Zhou Deng ${ }^{2 *}$ \\ and Xing-Quan Zhu* ${ }^{*}$
}

\begin{abstract}
Background: Toxoplasma gondii is a widely prevalent protozoan parasite that causes serious toxoplasmosis in humans and animals. The present study aimed to determine the genetic diversity of $T$. gondii isolates from pigs in Jiangxi, Sichuan, Guangdong Provinces and Chongqing Municipality in China using multilocous polymerase chain reaction-restriction fragment length polymorphism (PCR-RFLP) technology.

Methods: A total of 38 DNA samples were extracted from hilar lymph nodes of pigs with suspected toxoplasmosis, and were detected for the presence of $T$. gondii by semi-nested PCR of B1 gene. The positive DNA samples were typed at 11 genetic markers, including 10 nuclear loci, namely, SAG1, 5'-SAG2 and 3'-SAG2, alternative SAG2, SAG3, BTUB, GRA6, C22-8, c29-2, L358, PK1, and an apicoplast locus Apico.

Results: Twenty-five of the 38 DNA samples were T. gondii B1 gene positive. Complete genotyping data for all loci could be obtained for 17 of the 25 samples. Two genotypes were revealed (ToxoDB PCR-RFLP genotypes \#9 and \#3). Sixteen samples belong to genotype \#9 which is the major lineage in mainland China and one sample belongs to genotype \#3 which is Type II variant.

Conclusions: To our knowledge, this is the first report of genetic typing of T. gondii isolates from pigs in Jiangxi, Sichuan Province and Chongqing Municipality, and the first report of ToxoDB \#3 T. gondii from pigs in China. These results have implications for the prevention and control of foodborne toxoplasmosis in humans.
\end{abstract}

Keywords: Toxoplasma gondii, Toxoplasmosis, Genetic characterization, Pig, China

\section{Background}

Toxoplasma gondii is an obligate intracellular protozoan parasite, infecting all warm-blooded animals and human beings worldwide [1-5]. It is transmitted to humans through consumption of undercooked meat containing T. gondii tissue cysts or food or water contaminated by oocysts shed in the feces of infected cats. Toxoplasmosis is one of the main illnesses related to foodborne hospitalizations and deaths [6]. One third of the world population is chronically infected with this parasite [7]. Although T. gondii infection in most people appears to

\footnotetext{
*Correspondence: shzhdeng@163.com; xingquanzhu1@hotmail.com ${ }^{2}$ College of Animal Science and Technology, Jiangxi Agricultural University, Nanchang, Jiangxi Province 330045, PR China

Full list of author information is available at the end of the article
}

be asymptomatic, it may result in life-threatening illness in some immuno-compromised individuals [8].

In China, pigs are the primary livestock raised for human consumption, and they are commonly susceptible to T. gondii. This provides the parasite a potential route to transmit the infection via consumption of raw or undercooked meat. T. gondii may cause serious diseases such as blindness through consuming raw pork [9].

According to genotypic analysis based on multi-locus approaches, such as PCR-RFLP and microsatellite typing, T. gondii strains isolated from humans and animals in North America and Europe have been sorted into 3 clonal lineages, referred to as type I, II, and III [10-12]. A fourth clonal lineage (type 12) in North America was identified in wildlife recently [13]. However, T. gondii

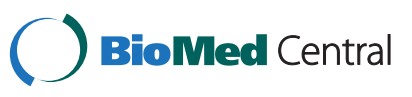


isolates from South America are characterized with higher genetic diversity [14-16].

China is a big country, but only limited reports concerning genetic characterization of $T$. gondii isolates from pigs are available [17-19]. Such information is still not available for some regions of China especially Jiangxi, Sichuan Provinces and Chongqing Municipality, where swine industry plays an important role in agricultural economy. Thus, the objective of this study is to better understand the genetic diversity of $T$. gondii isolates from pigs in these localities of China.

\section{Methods}

\section{Sample collection}

Hilar lymph nodes were collected, post slaughter, from 38 dead pigs (36 slaughter pigs and 2 sows) with suspected toxoplasmosis during May 2010-March 2013. No ethical approval is required. These pigs came from different geographic regions of China, including 33 from Jiangxi Province, 3 from Sichuan Province, 1 from Chongqing Municipality, and 1 from Guangdong Province, and they all exhibited typical symptoms of toxoplasmosis, which manifest primarily as high fever, dyspnea, subcutaneous hemorrhage, abortion, enlargement and necrosis of liver and spleen.

\section{Genomic DNA extraction}

Genomic DNA was extracted from these hilar lymph nodes using TIANamp Genomic DNA kit (TianGen ${ }^{\mathrm{ru}}$, Beijing, China) according to manufacturer's recommendations. In brief, $30 \mathrm{mg}$ of the hilar lymph nodes were treated with sodium dodecyl sulphate/proteinase $\mathrm{K}$ at $56^{\circ} \mathrm{C}$ for overnight digestion in a thermostat water bath. DNA samples were prepared after purification by silica gel column chromatography and eluted into $50 \mu \mathrm{l}$ elution buffer. Then, a semi-nested PCR was performed to detect the $T$. gondii B1 gene following the previously described protocol [20].

\section{Genetic characterization of $T$. gondii isolates}

Multilocous polymerase chain reaction-restriction fragment length polymorphism (PCR-RFLP) method [21] was employed to genetically characterize the $T$. gondii isolates from pigs. Firstly, pre-amplification was carried out using a set of mixed external primers in a single reaction. Then $1 \mu \mathrm{l}$ of the products served as template DNA for nested PCR with internal primers for each marker, respectively. The nested PCR products were digested with restriction endonucleases. The restriction fragments were resolved in $2.5 \%-3 \%$ agarose gel to display single nucleotide polymorphisms (SNPs) using a gel document system (UVP GelDoc-It ${ }^{\mathrm{tm}}$ Imaging System, Cambridge, U.K.), and the genotypes of T. gondii isolates were finally revealed.

\section{Results and discussion}

Twenty-five of the 38 DNA samples were $T$. gondii B1 gene positive, including 23 from slaughter pigs and 2 from sows. Due to low DNA concentration, only 17 DNA samples presented complete genotyping data. The results of genotyping of these isolates and 9 references were summarized in Table 1 . Two genotypes were revealed (ToxoDB PCR-RFLP genotypes \#9 and \#3). It is interesting that only one genotype (ToxoDB\#9) was identified from all $12 \mathrm{~T}$. gondii isolates from different districts of Jiangxi Province, which suggests that this genotype is predominantly prevalent in Jiangxi Province and the genetic diversity of $T$. gondii may be low in pigs in this province, although further studies using more samples collected from much broader geographical localities of this province are warranted before a valid conclusion can be drawn. Moreover, ToxoDB\#9 was also identified in 4 isolates from 3 localities (Zigong and Ziyang in Sichuan and Rongchang in Chongqing) near the border of Sichuan Province and Chongqing Municipality. This same genotype was previously identified in cats in Beijing Municipality, Guangdong and Anhui Provinces [22-24], and it was also founded in pigs in Guangdong, Henan, Yunnan and Anhui Provinces [17-19,25]. Based on these data, ToxoDB\#9 was a predominant lineage prevalent in Mainland China. Interestingly, ToxoDB\#9 has been identified in North and Latin America [15,26-28], as well as other Asian countries, such as Sri Lanka, Vietnam $[29,30]$, indicating that it has a worldwide distribution. In the near future, it would be interesting to genotype $T$. gondii isolates from humans in the same localities to see if humans and animals share the same genotypes.

In this study, another genotype ToxoDB\#3 (the type II variant) was identified in a pig in Zhongshan, Guangdong Province. This was the fourth time that ToxoDB\#3 was identified in China. Previously, this type was founded from sheep in Qinghai Province [17], from birds in Xinjiang Uygur Autonomous Region [31] and from sparrow in Lanzhou, Gansu Province [32]. This is also the first report of ToxoDB\#3 from pigs in China, which indicated that ToxoDB\#3 is also a major lineage prevalent in Mainland China.

For Chinese people especially the Han ethnic, pork is the main meat of choice. With the development of the economy in China, the standard of life has greatly improved in recent years. As a result, the pig industry and pork products have been driven by increasing consumption for high quality animal protein. Unlike herbivorous cattle and sheep, pigs are omnivores, they feed on a variety of animal meat and vegetable matter, which increases the chance of contact with cat feces and exposure to T. gondii, especially for those free-range pigs. Thus, pigs pose a risk for transmission of toxoplasmosis to human beings. 
Table 1 Summary of genotyping of Toxoplasma gondii isolates from pigs in different geographic regions of China

\begin{tabular}{|c|c|c|c|c|c|c|c|c|c|c|c|c|c|c|}
\hline Isolate ID & Host & Location & SAG1 & $\begin{array}{l}5^{\prime}+3^{\prime} \\
\text { SAG2 }\end{array}$ & $\begin{array}{l}\text { Alternative } \\
\text { SAG2 }\end{array}$ & SAG3 & BTUB & GRA6 & $\begin{array}{l}\text { c22- } \\
8\end{array}$ & $\begin{array}{l}\text { c29- } \\
2\end{array}$ & L358 & PK1 & Apico & Genotype \\
\hline GT1 & Goat & United States & I & I & 1 & I & I & I & I & I & I & I & I & $\begin{array}{l}\text { Reference, Type I, } \\
\text { ToxoDB \#10 }\end{array}$ \\
\hline PTG & Sheep & United States & $\|/\|$ & $\|$ & $\|$ & $\|$ & $\|$ & $\|$ & $\|$ & $\|$ & $\|$ & $\|$ & । & $\begin{array}{l}\text { Reference, Type II, } \\
\text { ToxoDB \#1 }\end{array}$ \\
\hline $\mathrm{CTG}$ & Cat & United States & $\|/\|$ & III & III & III & III & III & III & III & III & III & I & $\begin{array}{l}\text { Reference, Type III, } \\
\text { ToxoDB \#2 }\end{array}$ \\
\hline MAS & Human & France & $u-1$ & I & $\|$ & III & III & III & $u-1$ & । & I & III & I & $\begin{array}{l}\text { Reference, ToxoDB } \\
\# 17\end{array}$ \\
\hline TgCgCa1 & Cougar & Canada & । & । & $\|$ & III & III & $\|$ & $\|$ & $u-1$ & । & $u-2$ & । & $\begin{array}{l}\text { Reference, ToxoDB } \\
\# 66\end{array}$ \\
\hline TgCatBr5 & Cat & Brazil & । & III & III & III & $\|$ & III & । & । & । & $u-1$ & 1 & $\begin{array}{l}\text { Reference, ToxoDB } \\
\# 19\end{array}$ \\
\hline TgWtdSc40 & WTD & United States & $u-1$ & $\|$ & $\|$ & $\|$ & $\|$ & $\|$ & $\|$ & $\|$ & I & $\|$ & I & $\begin{array}{l}\text { Reference, Type 12, } \\
\text { ToxoDB \#5 }\end{array}$ \\
\hline TgCatBr64 & Cat & Brazil & । & I & $u-1$ & III & III & III & $u-1$ & I & III & III & I & $\begin{array}{l}\text { Reference, ToxoDB } \\
\# 111\end{array}$ \\
\hline TgRsCr1 & Toucan & Costa Rica & $u-1$ & I & $\|$ & III & I & III & $u-2$ & I & I & III & I & $\begin{array}{l}\text { Reference, ToxoDB } \\
\# 52\end{array}$ \\
\hline TgPSZ30 & $\mathrm{SP}$ & Zigong, Sichuan & $u-1$ & $\|$ & $\|$ & III & III & $\|$ & $\|$ & III & $\|$ & $\|$ & I & ToxoDB \#9 \\
\hline TgPSZ41 & SP & Zigong, Sichuan & $u-1$ & $\|$ & $\|$ & III & III & $\|$ & $\|$ & III & $\|$ & $\|$ & I & ToxoDB \#9 \\
\hline TgPSY19 & $\mathrm{SP}$ & Ziyang, Sichuan & $u-1$ & $\|$ & $\|$ & III & III & $\|$ & $\|$ & III & $\|$ & $\|$ & I & ToxoDB \#9 \\
\hline TgPCR51 & $\mathrm{SP}$ & $\begin{array}{l}\text { Rongchang, } \\
\text { Chongqing }\end{array}$ & $u-1$ & $\|$ & $\|$ & III & III & $\|$ & $\|$ & III & $\|$ & $\|$ & I & ToxoDB \#9 \\
\hline TgPJX1 & Sow & $\begin{array}{l}\text { Wannian, } \\
\text { Jiangxi }\end{array}$ & $u-1$ & $\|$ & $\|$ & III & III & $\|$ & $\|$ & III & $\|$ & $\|$ & 1 & ToxoDB \#9 \\
\hline TgPJX2 & $\mathrm{SP}$ & Xinjian, Jiangxi & $u-1$ & $\|$ & $\|$ & III & III & $\|$ & $\|$ & III & $\|$ & $\|$ & । & ToxoDB \#9 \\
\hline TgPJX3 & $\mathrm{SP}$ & $\begin{array}{l}\text { Nanchang, } \\
\text { Jiangxi }\end{array}$ & $u-1$ & $\|$ & $\|$ & III & III & $\|$ & $\|$ & III & $\|$ & $\|$ & 1 & ToxoDB \#9 \\
\hline TgPJX4 & Sow & $\begin{array}{l}\text { Xingguo, } \\
\text { Jiangxi }\end{array}$ & $u-1$ & $\|$ & $\|$ & III & III & $\|$ & $\|$ & III & $\|$ & $\|$ & I & ToxoDB \#9 \\
\hline TgPJX5 & $\mathrm{SP}$ & $\begin{array}{l}\text { Xingguo, } \\
\text { Jiangxi }\end{array}$ & $u-1$ & $\|$ & $\|$ & III & III & $\|$ & $\|$ & III & $\|$ & $\|$ & I & ToxoDB \#9 \\
\hline TgPJX6 & $\mathrm{SP}$ & $\begin{array}{l}\text { Nanchang, } \\
\text { Jiangxi }\end{array}$ & $u-1$ & $\|$ & $\|$ & III & III & $\|$ & $\|$ & III & $\|$ & $\|$ & I & ToxoDB \#9 \\
\hline TgPJX7 & $\mathrm{SP}$ & Yujiang, Jiangxi & $u-1$ & $\|$ & $\|$ & III & III & $\|$ & $\|$ & III & $\|$ & $\|$ & I & ToxoDB \#9 \\
\hline TgPJX8 & $\mathrm{SP}$ & Yujiang, Jiangxi & $u-1$ & $\|$ & $\|$ & III & III & $\|$ & $\|$ & III & $\|$ & $\|$ & I & ToxoDB \#9 \\
\hline TgPJX9 & $\mathrm{SP}$ & Yujiang, Jiangxi & $u-1$ & $\|$ & $\|$ & III & III & $\|$ & $\|$ & III & $\|$ & $\|$ & 1 & ToxoDB \#9 \\
\hline TgPJX10 & $\mathrm{SP}$ & Yujiang, Jiangxi & $u-1$ & $\|$ & $\|$ & III & III & $\|$ & $\|$ & III & $\|$ & $\|$ & I & ToxoDB \#9 \\
\hline TgPJX11 & $\mathrm{SP}$ & Yujiang, Jiangxi & $u-1$ & $\|$ & $\|$ & III & III & $\|$ & $\|$ & III & $\|$ & $\|$ & I & ToxoDB \#9 \\
\hline TgPJX12 & SP & $\begin{array}{l}\text { Nanchang, } \\
\text { Jiangxi }\end{array}$ & $u-1$ & $\|$ & $\|$ & III & III & $\|$ & $\|$ & III & $\|$ & $\|$ & I & ToxoDB \#9 \\
\hline TgPZS1 & $\mathrm{SP}$ & $\begin{array}{l}\text { Zhongshan, } \\
\text { Guangdong }\end{array}$ & $\|$ & $\|$ & $\|$ & $\|$ & $\|$ & $\|$ & $\|$ & $\|$ & $\|$ & $\|$ & I & ToxoDB \#3 \\
\hline
\end{tabular}

$\mathrm{u}-1$ and $\mathrm{u}-2^{*}$ represent unique RFLP genotypes, respectively. WTD White-tailed Deer.

SP Slaughter pig.

\section{Conclusions}

The present study has genetically characterized $T$. gondii isolates from pigs in Jiangxi, Sichuan, Guangdong Provinces and Chongqing Municipality. Two genotypes were revealed (ToxoDB PCR-RFLP genotypes \#9 and \#3), with the genotype \#9 as the major lineage in mainland China. To our knowledge, this is the first report of genetic typing of T. gondii isolates from pigs in these localities, and the first report of ToxoDB genotype \#3 from pigs in China. These findings not only enrich genetic diversity 
of $T$. gondii, but also have implications for the prevention and control of foodborne toxoplasmosis in humans.

\section{Competing interests}

The authors declare that they have no competing interests.

\section{Authors' contributions}

XQZ and CS conceived and designed the study, and critically revised the manuscript. HHJ, SYH and SZD performed the experiments, analyzed the data and drafted the manuscript. DHZ and XXZ helped in study design, study implementation and manuscript revision. All authors read and approved the final manuscript.

\section{Acknowledgements}

Project support was provided by the National Natural Science Foundation of China (Grant Nos. 31228022, 31101812,31230073 and 31172316), and the Science Fund for Creative Research Groups of Gansu Province (Grant No. 1210RJIA006).

\section{Author details}

'State Key Laboratory of Veterinary Etiological Biology, Key Laboratory of Veterinary Parasitology of Gansu Province, Lanzhou Veterinary Research Institute, Chinese Academy of Agricultural Sciences, Lanzhou, Gansu Province 730046, PR China. ${ }^{2}$ College of Animal Science and Technology, Jiangxi Agricultural University, Nanchang, Jiangxi Province 330045, PR China. ${ }^{3}$ Department of Microbiology, The University of Tennessee, Knoxville, TN 37996, USA. ${ }^{4}$ College of Agriculture, Yanbian University, Yanji, Jilin Province 133002, PR China.

Received: 14 June 2013 Accepted: 1 August 2013

Published: 7 August 2013

\section{References}

1. Dubey JP: Toxoplasmosis of animals and humans. 2nd edition. Boca Raton, Florida: CRC Press; 2010:313.

2. Nardoni S, Angelici MC, Mugnaini L, Mancianti F: Prevalence of Toxoplasma gondii infection in Myocastor coypus in a protected Italian wetland. Parasit Vectors 2011, 4:240.

3. Chen J, Xu MJ, Zhou DH, Song HQ, Wang CR, Zhu XQ: Canine and feline parasitic zoonoses in China. Parasit Vectors 2012, 5:152.

4. Tian YM, Dai FY, Huang SY, Deng ZH, Duan G, Zhou DH, Yang JF, Weng YB, Zhu XQ, Zou FC: First report of Toxoplasma gondii seroprevalence in peafowls in Yunnan Province, Southwestern China. Parasit Vectors 2012, 5:205.

5. Zhou P, Chen Z, Li HL, Zheng H, He S, Lin RQ, Zhu XQ: Toxoplasma gondii infection in humans in China. Parasit Vectors 2011, 4:165.

6. Dubey JP: Toxoplasmosis-a waterborne zoonosis. Vet Parasitol 2004, 126:57-72.

7. Weiss LM, Dubey JP: Toxoplasmosis: a history of clinical observations. Int $J$ Parasitol 2009, 39:895-901.

8. Montoya JG, Liesenfeld O: Toxoplasmosis. Lancet 2004, 363:1965-1976.

9. Choi WY, Nam HW, Kwak NH, Huh W, Kim YR, Kang MW, Cho SY, Dubey JP: Foodborne outbreaks of human toxoplasmosis. J Infect Dis 1997, 175:1280-1282.

10. Ajzenberg D, Banuls AL, Tibayrenc M, Darde ML: Microsatellite analysis of Toxoplasma gondii shows considerable polymorphism structured into two main clonal groups. Int I Parasitol 2002, 32:27-38.

11. Darde ML, Bouteille B, Pestre-Alexandre M: Isoenzyme analysis of 35 Toxoplasma gondii isolates and the biological and epidemiological implications. J Parasitol 1992, 78:786-794.

12. Howe DK, Sibley LD: Toxoplasma gondii comprises three clonal lineages: correlation of parasite genotype with human disease. J Infect Dis 1995, 172:1561-1566.

13. Khan A, Dubey JP, Su C, Ajioka JW, Rosenthal BM, Sibley LD: Genetic analyses of atypical Toxoplasma gondii strains reveal a fourth clonal lineage in North America. Int J Parasitol 2011, 41:645-655.

14. Dubey JP, Graham DH, Blackston CR, Lehmann T, Gennari SM, Ragozo AM, Nishi SM, Shen SK, Kwok OC, Hill DE, Thulliez P: Biological and genetic characterisation of Toxoplasma gondii isolates from chickens (Gallus domesticus) from Sao Paulo, Brazil: unexpected findings. Int I Parasitol 2002, 32:99-105.
15. Dubey JP, Sundar N, Hill D, Velmurugan GV, Bandini LA, Kwok OC, Majumdar D, Su C: High prevalence and abundant atypical genotypes of Toxoplasma gondii isolated from lambs destined for human consumption in the USA. Int J Parasitol 2008, 38:999-1006.

16. Lehmann T, Marcet PL, Graham DH, Dahl ER, Dubey JP: Globalization and the population structure of Toxoplasma gondii. Proc Natl Acad Sci USA 2006, 103:11423-11428.

17. Zhou P, Zhang H, Lin RQ, Zhang DL, Song HQ, Su C, Zhu XQ: Genetic characterization of Toxoplasma gondii isolates from China. Parasitol Int 2009, 58:193-195.

18. Zhou P, Nie H, Zhang LX, Wang HY, Yin CC, Su C, Zhu XQ, Zhao JL: Genetic characterization of Toxoplasma gondii isolates from pigs in China. J Parasitol 2010, 96:1027-1029.

19. Zhou P, Sun XT, Yin CC, Yang JF, Yuan ZG, Yan HK, Zhu XQ, Zou FC: Genetic characterization of Toxoplasma gondii isolates from pigs in southwestern China. J Parasitol 2011, 97:1193-1195.

20. Hill DE, Chirukandoth S, Dubey JP, Lunney JK, Gamble HR: Comparison of detection methods for Toxoplasma gondii in naturally and experimentally infected swine. Vet Parasitol 2006, 141:9-17.

21. Su C, Shwab EK, Zhou P, Zhu XQ, Dubey JP: Moving towards an integrated approach to molecular detection and identification of Toxoplasma gondii. Parasitology 2010, 137:1-11.

22. Dubey JP, Zhu XQ, Sundar N, Zhang H, Kwok OC, Su C: Genetic and biologic characterization of Toxoplasma gondii isolates of cats from China. Vet Parasitol 2007, 145:352-356.

23. Qian W, Wang H, Su C, Shan D, Cui X, Yang N, Lv C, Liu Q: Isolation and characterization of Toxoplasma gondii strains from stray cats revealed a single genotype in Beijing, China. Vet Parasitol 2012, 187:408-413.

24. Chen ZW, Gao JM, Huo XX, Wang L, Yu L, Halm-Lai F, Xu YH, Song WJ, Hide G, Shen JL, Lun ZR: Genotyping of Toxoplasma gondii isolates from cats in different geographic regions of China. Vet Parasitol 2011, 183:166-170.

25. Wang H, Wang T, Luo Q, Huo X, Wang L, Liu T, Xu X, Wang Y, Lu F, Lun Z, Yu L, Shen J: Prevalence and genotypes of Toxoplasma gondii in pork from retail meat stores in Eastern China. Int J Food Microbiol 2012, 157:393-397.

26. Dubey JP, Velmurugan GV, Alvarado-Esquivel C, Alvarado-Esquivel D, Rodriguez-Pena S, Martinez-Garcia S, Gonzalez-Herrera A, Ferreira LR, Kwok OC, Su C: Isolation of Toxoplasma gondii from animals in Durango, Mexico. J Parasitol 2009, 95:319-322.

27. Dubey JP, Cortes-Vecino JA, Vargas-Duarte JJ, Sundar N, Velmurugan GV, Bandini LM, Polo L, Zambrano L, Mora LE, Kwok OC, Smith T, Su C: Prevalence of Toxoplasma gondii in dogs from Colombia, South America and genetic characterization of T. gondii isolates. Vet Parasitol 2007, 145:45-50.

28. Dubey JP, Sundar N, Gennari SM, Minervino AH, Farias NA, Ruas JL, Dos Santos TR, Cavalcante GT, Kwok OC, Su C: Biologic and genetic comparison of Toxoplasma gondii isolates in free-range chickens from the northern Para state and the southern state Rio Grande do Sul, Brazil revealed highly diverse and distinct parasite populations. Vet Parasitol 2007, 143:182-188.

29. Dubey JP, Huong LT, Sundar N, Su C: Genetic characterization of Toxoplasma gondii isolates in dogs from Vietnam suggests their South American origin. Vet Parasitol 2007, 146:347-351.

30. Dubey JP, Rajapakse RP, Wijesundera RR, Sundar N, Velmurugan GV, Kwok OC, Su C: Prevalence of Toxoplasma gondii in dogs from Sri Lanka and genetic characterization of the parasite isolates. Vet Parasitol 2007, 146:341-346.

31. Huang SY, Cong W, Zhou P, Zhou DH, Wu SM, Xu MJ, Zou FC, Song HQ, Zhu XQ: First report of genotyping of Toxoplasma gondii isolates from wild birds in China. J Parasitol 2012, 98:681-682.

32. Cong W, Huang SY, Zhou DH, Zhang XX, Zhang NZ, Zhao Q, Zhu XQ: Prevalence and genetic characterization of Toxoplasma gondii in house sparrows (Passer domesticus) in Lanzhou, China. Korean J Parasitol 2013, 51:363-367.

\section{doi:10.1186/1756-3305-6-227}

Cite this article as: Jiang et al:: Genetic characterization of Toxoplasma gondii from pigs from different localities in China by PCR-RFLP. Parasites \& Vectors 2013 6:227. 